\title{
Efecto de Escherichia coli y Salmonella spp en el crecimiento y mortalidad de crías de alpacas (Vicugna pacos)
}

\author{
Effect of $E$. coli and Salmonella spp on the growth and mortality \\ of baby alpacas (Vicugna pacos)
}

\author{
Víctor Carhuapoma $D^{1,3}$, Nicasio Valencia M. ${ }^{2}$, Rufino Paucar C. ${ }^{2}$, Paul H. Mayhua \\ M. $^{2}$, Rodrigo Huamán J. ${ }^{2}$, Epifanio Lizana-Hilario ${ }^{2}$
}

\section{Resumen}

El estudio tuvo como objetivo evaluar el efecto de Escherichia coli y Salmonella spp en el crecimiento y mortalidad de crías de alpacas (Vicugna pacos). Se seleccionaron 104 crías de alpacas Huacaya con procesos diarreicos y con edad promedio de $10.0 \pm 2$ días, peso de $9.6 \pm 0.5 \mathrm{~kg}$ y altura a la cruz de $85.0 \pm 0.5 \mathrm{~cm}$. Se tomaron muestras de hisopados rectales y se cultivaron en medios de cultivo selectivos de agares MacConkey, EMB, SS, XHD y BIH, y se hicieron pruebas bioquímicas. Con base a los resultados positivos a E. coli y Salmonella spp se seleccionaron 80 crías que fueron distribuidas en cuatro grupos: G1, E. coli (n=23); G2, Salmonella spp (n=17); G3, E. coli-Salmonella spp $(n=12)$; $\mathrm{G} 4$, Testigo $(\mathrm{n}=28)$, positivos a uno o a los dos gérmenes y tratados con tetraciclinas. Los animales fueron pesados y medidos (altura a la cruz) a las 4 y 8 semanas del estudio y la mortalidad fue registrada. El peso y la talla corporal fueron más afectados en G1, G2 y G3 que en G4 ( $\mathrm{p}<0.01$ ). Asimismo, la tasa de mortalidad fue mayor en G1, G2 y G3 que en G4 para ambos sexos. Salmonella spp y E. coli se evidenciaron como agentes patógenos negativos para el crecimiento y mortalidad en crías de alpacas con diarrea.

Palabras clave: alpaca; diarrea; crecimiento; mortalidad

\section{Abstract}

The aim of this study was to evaluate the effect of E. coli and Salmonella spp on the growth and mortality of baby alpacas (Vicugna pacos). It was selected 104 Huacaya alpacas with diarrheal processes and with an average age of $10.0 \pm 2$ days, body weight of $9.6 \pm 0.5 \mathrm{~kg}$ and height at the withers of $85.0 \pm 0.5 \mathrm{~cm}$. Samples of rectal swabs were

\footnotetext{
${ }^{1}$ Centro de Investigación Científica Multidisciplinaria de Ingeniería, Escuela Profesional de Zootecnia, Universidad Nacional de Huancavelica, Perú

${ }^{2}$ Escuela Profesional de Zootecnia, Universidad Nacional de Huancavelica, Perú

${ }^{3}$ E-mail: yachayruacc@hotmail.com
}

Recibido: 1 de marzo de 2018

Aceptado para publicación: 26 de febrero de 2019 
cultured in selective culture media of MacConkey, EMB, SS, XHD and BIH agar, and biochemical tests were performed. Based on the positive results of E. coli and Salmonella spp. A total of 80 animals were selected and assigned into four groups: $\mathrm{G} 1, E$. coli $(\mathrm{n}=23)$; G2, Salmonella spp ( $\mathrm{n}=17)$; G3, E. coli - Salmonella $\operatorname{spp}(\mathrm{n}=12)$; G4, control ( $\mathrm{n}=28)$, positive for one or both germs and treated with tetracycline. The animals were weighed and measured (height at the withers) at weeks 4 and 8 of the study and mortality was recorded. The weight and corporal height were more affected in G1, G2 and G3 that the G4 $(\mathrm{p}<0.01)$. Likewise, the rate of mortality was major in G1, G2 and G3 and in G4 in both sexes. Salmonella spp and E. coli affected the growth and caused mortality in baby alpacas with diarrhoea.

Key words: alpaca; diarrhoea; increase; mortality

\section{INTRODUCCIÓN}

Las elevadas tasas de mortalidad por causas infecciosas en las al pacas (Vicugna pacos), especialmente en sus crías, son uno de los factores limitantes para el desarrollo económico de las actividades pecuarias en la zona altoandina (Rosadio et al., 2012b). Las pérdidas de crías de alpacas por causas de enteritis infecciosa bacteriana se generan dentro de los primeros cuatro meses de vida, alcanzando cifras que superan el $50 \%$ de mortalidad (Siuce et al., 2015); donde Clostridium perfringens, Escherichia coli enteropatógena y Salmonella spp se encuentran usualmente involucradas como agentes causales en el denominado complejo bacterodiarreico neonatal (Mamani y Huanca, 2011).

Este síndrome cursa con disfunción intestinal, usualmente asociado a diarrea, debido a la sobre estimulación de la función secretora de las criptas epiteliales (salmonelosis, colibacilosis), incremento de la permeabilidad (enteritis clostridial) e hipermotilidad (Siuce et al., 2015). La salmonelosis en la alpaca no ha sido muy estudiada, pero los reportes existentes permiten presumir que Salmonella spp estaría asociada a la enteritis infecciosa bacteriana (Oha, 2012).
Estos agentes bacterianos generan un impacto económico negativo al productor, impidiendo el crecimiento sostenido de la actividad alpaquera al disminuir la producción neta de animales y la cantidad de animales que contribuyan con la variabilidad genética. Los casos clínicos en las crías de alpacas se presentan con deshidratación progresiva, acidosis, postración, pérdida de peso y muerte (Ortiz, 2011). No obstante, no se dispone de trabajos científicos que determinen su nivel de participación en el complejo diarreico neonatal con respecto a los parámetros productivos (crecimiento, ganancia de peso vivo, supervivencia) de la alpaca.

El objetivo del presente estudio fue evaluar el efecto de E. coli y Salmonella spp sobre el crecimiento y mortalidad de crías de alpacas. Asimismo, identificar las bacterias enteropatógenas presentes.

\section{Materiales y Métodos}

El estudio se realizó en el predio «Rumi Huasi» del sector de Huamanrazu de la Comunidad Campesina de Santa Bárbara, en Huancavelica, Perú. La zona está situada entre 4200 y $4500 \mathrm{msnm}$, con temperatura promedio anual de $5.6{ }^{\circ} \mathrm{C}$. Los estudios bacteriológicos se realizaron en el Laborato- 
Cuadro 1. Crías de alpacas con enteropatías diarreicas positivos a Escherichia coli, Salmonella spp y E. coli - Salmonela $\operatorname{spp}(\mathrm{n}=104)$

\begin{tabular}{lcccc}
\hline \multirow{2}{*}{ Patógeno } & \multicolumn{2}{c}{ Machos } & \multicolumn{2}{c}{ Hembras } \\
\cline { 2 - 5 } & $\mathrm{n}$ & $\%$ & $\mathrm{n}$ & $\%$ \\
\hline $\begin{array}{l}\text { Escherichia } \\
\text { coli }\end{array}$ & 19 & 12.2 & 18 & 11.6 \\
$\begin{array}{l}\text { Salmonella } \\
\text { spp }\end{array}$ & 18 & 11.6 & 17 & 11.0 \\
$\begin{array}{l}\text { E. coli- } \\
\text { Salmonella } \\
\text { spp }\end{array}$ & 16 & 10.3 & 16 & 10.3 \\
\hline
\end{tabular}

rio Central de Investigación, Área de Salud Animal, de la Universidad Nacional de Huancavelica. La fase de campo tuvo una duración de dos meses y se realizó durante las épocas de lluvias (febrero-marzo). Se seleccionaron 104 crías de alpacas Huacaya, sin distinción de sexo, con edad promedio de $10.0 \pm 2$ días, peso de $9.6 \pm 0.5 \mathrm{~kg}$ y altura a la cruz de $85.0 \pm 0.5 \mathrm{~cm}$. Todos los animales seleccionados ingresaron al estudio preliminar con cuadros clínicos de diarrea entre 3 y 8 días.

Se recolectaron muestras de heces por triplicado de las 104 crías. Las muestras fueron tomadas por el método de hisopado rectal y suspendidas en medio de transporte Stuart. Se hizo un pool con las tres muestras de cada animal, las cuales fueron cultivadas en medios selectivos de agar: MacConkey y eosina azul de metileno (EMB) para E. coli y Salmonella-Shigella (SS), xilosa lisina desoxicolato XHD para Salmonella spp y enriquecidas con caldo de infusión cerebro corazón (BIH). Para la identificación y diferenciación de las cepas se realizó la caracterización morfológica (forma elevación, margen) y microscópica (coloración Gram), así como pruebas bioquímicas convencionales (agar-hierro-triple azúcar [TSI], agar de hierro de lisina [LIA], sulfuro-indol-motilidad [SIM] y catalasa).

Con base a los resultados de laboratorio, se seleccionaron las crías positivas a $E$. coli, Salmonella spp y E. coli - Salmonella spp ( $\mathrm{n}=104$ crías; Cuadro 1) a las 72 horas de la identificación bacteriológica. Para la evaluación de peso y talla solo se consideraron los animales que sobrevivieron al proceso diarreico, quedando los siguientes grupos: G1, E. coli (n=23 [13 machos y 10 hembras]); G2, Salmonella spp ( $\mathrm{n}=17$ [9 machos y 8 hembras]); G3, E. coli y Salmonella spp ( $\mathrm{n}=12$ [6 machos y 6 hembras]); G4, Testigo tratado, E. coli, Salmonella spp y E. coli y Salmonella $\mathrm{spp}(\mathrm{n}=28$ [14 machos y 14 hembras]).

Todos los animales fueron desparasitados con un anticoccidiostático (Toltrazuril) y puestos en cuarentena en potreros reservados para este propósito al inicio del estudio. Los animales del grupo testigo (G4) fueron tratados con tetraciclinas vía i. $\mathrm{m}$. a dosis $1 \mathrm{ml} / 10 \mathrm{~kg}$ de peso vivo durante tres semanas con el fin de controlar la diarrea.

El peso, talla corporal (altura a la cruz) y mortalidad fueron registrados semanalmente y por ocho semanas. Las mediciones de campo se hicieron en las mañanas utilizando una balanza circular de $100 \mathrm{~kg}$ de capacidad y una cinta métrica. El porcentaje de mortalidad se calculó dividiendo el número de crías muertas entre el total de crías, expresado en forma porcentual. Para las evaluaciones de peso y talla corporal se consideraron a los animales que sobrevivieron durante el estudio. El protocolo fue certificado por el Comité de Ética de Educación y Ciencias de la Salud para la Investigación de la Universidad Nacional de Huancavelica con resolución N . $^{\circ}$ 0724-2015-CECSI-UNH. Los datos fueron analizados mediante el análisis de varianza y la prueba de Tukey $(\mathrm{p}<0.05)$, utilizando el programa SPSS v. 20. 
Cuadro 2. Peso corporal ( $\mathrm{kg})$ de 80 crías de alpacas $^{1}$ con enteropatías producidas por $E$. coli y Salmonella spp (medias y desviación estándar)

\begin{tabular}{lcccccc}
\hline \multirow{2}{*}{ Grupos } & \multicolumn{4}{c}{ Hembras } & \multicolumn{3}{c}{ Machos } \\
\cline { 2 - 7 } & Inicial & 4 semanas & 8 semanas & Inicial & 4 semanas & 8 semana \\
\hline G1 - E. coli & $9.6 \pm 1.0^{\mathrm{a}}$ & $11.3 \pm 1.2^{\mathrm{a}}$ & $14.3 \pm 1.4^{\mathrm{b}}$ & $9.9 \pm 1.2^{\mathrm{a}}$ & $11.7 \pm 1.3^{\mathrm{a}}$ & $16.2 \pm 1.3$ \\
G2 - Salmonella & $9.5 \pm 2.0^{\mathrm{a}}$ & $10.2 \pm 1.4^{\mathrm{a}}$ & $13.4 \pm 1.5^{\mathrm{b}}$ & $10.2 \pm 1.3^{\mathrm{a}}$ & $12.4 \pm 1.3^{\mathrm{a}}$ & $14.6 \pm 1.2$ \\
G3 - E. coli- & $9.2 \pm 1.8^{\mathrm{a}}$ & $11.2 \pm 1.6^{\mathrm{a}}$ & $12.6 \pm 1.4^{\mathrm{b}}$ & $9.9 \pm 1.4^{\mathrm{a}}$ & $11.9 \pm 1.2^{\mathrm{a}}$ & $13.4 \pm 1.4$ \\
$\quad$ Salmonella & & & & & \\
G4 - Testigo & $9.4 \pm 1.8^{\mathrm{a}}$ & $13.2 \pm 1.5^{\mathrm{b}}$ & $16.7 \pm 1.4^{\mathrm{c}}$ & $9.8 \pm 1.4^{\mathrm{a}}$ & $14.8 \pm 1.2^{\mathrm{b}}$ & $18.4 \pm 1.4$ \\
\hline a,b,c,d Superíndices diferentes dentro y entre columnas indican diferencia estadística $(\mathrm{p}<0.01)$ \\
${ }^{1}$ 23 crías en G1, 17 en G2, 12 en G3 y 28 en G4 $^{\text {2 Positivas a E. coli, Salmonella o ambas y tratadas con tetraciclinas }}$
\end{tabular}

Cuadro 3. Talla corporal (altura a la cruz) de 80 crías de alpacas $^{1}$ con enteropatías producidas por E. coli y Salmonella spp (medias y desviación estándar)

\begin{tabular}{lcccccc}
\hline \multirow{2}{*}{ Grupos } & \multicolumn{4}{c}{ Hembras } & \multicolumn{3}{c}{ Machos } \\
\cline { 2 - 7 } & Inicial & 4 semanas & 8 semanas & Inicial & 4 semanas & 8 semanas \\
\hline G1 - E. coli & $59.0 \pm 1.3^{\mathrm{a}}$ & $64.0 \pm 1.2^{\mathrm{a}}$ & $70.0 \pm 1.1^{\mathrm{b}}$ & $60.0 \pm 1.1^{\mathrm{a}}$ & $65.0 \pm 1.2^{\mathrm{a}}$ & $71.0 \pm 1.3^{\mathrm{b}}$ \\
G2 - Salmonella & $60.0 \pm 1.4^{\mathrm{a}}$ & $65.0 \pm 1.4^{\mathrm{b}}$ & $68.0 \pm 1.5^{\mathrm{a}}$ & $61.6 \pm 1.3^{\mathrm{a}}$ & $66.0 \pm 1.3^{\mathrm{b}}$ & $69.0 \pm 1.2^{\mathrm{a}}$ \\
G3 - E. coli - & $58.0 \pm 1.2^{\mathrm{a}}$ & $61.0 \pm 1.3^{\mathrm{c}}$ & $67.0 \pm 1.4^{\mathrm{c}}$ & $62.0 \pm 1.4^{\mathrm{a}}$ & $66.0 \pm 1.2^{\mathrm{c}}$ & $68.0 \pm 1.1^{\mathrm{c}}$ \\
$\quad$ Salmonella & & & & & & \\
G4 - Testigo ${ }^{2}$ & $61.0 \pm 1.0^{\mathrm{a}}$ & $72.0 \pm 1.2^{\mathrm{d}}$ & $85.0 \pm 1.1^{\mathrm{d}}$ & $63.0 \pm 1.2^{\mathrm{a}}$ & $78.0 \pm 1.2^{\mathrm{d}}$ & $84.0 \pm 1.1^{\mathrm{d}}$ \\
\hline${ }_{\mathrm{a}, \mathrm{b}, \mathrm{c}, \mathrm{d}}$ Superíndices diferentes dentro y entre de columnas indican diferencia estadística $(\mathrm{p}<0.01)$ \\
${ }^{1}$ 23 crías en G1, 17 en G2, 12 en G3 y 28 en G4 \\
${ }^{2}$ Positivas a E. coli, Salmonella o ambas y tratadas con tetraciclinas
\end{tabular}

\section{Resultados}

Las crías de los grupos G1, G2 y G3 ganaron menor peso que aquellas del grupo G4 (testigo) durante las 8 semanas del estudio, tanto en hembras como en machos ( $<<0.01$; Cuadro 2), siendo la asociación $E$. coli - Salmonella spp, las que causaron un mayor efecto negativo en el peso corporal. Similar tendencia fue observada a las cuatro semanas de evaluación en ambos sexos.
La talla de las crías de los grupos G1, G2 y G3 a las ocho semanas fue menor en comparación con las crías de G4 (testigo) en ambos sexos ( $<<0.01$; Cuadro 3). Similar tendencia fue observada a las cuatro semanas de evaluación. El porcentaje de mortalidad fue significativamente mayor en G1, G2 y G3 con relación a G4 (testigo) en ambos sexos (Cuadro 4). 
Cuadro 4. Mortalidad (\%) en 104 crías $^{1}$ de alpacas con enteropatías producidas por Escherichia coli y Salmonella spp

\begin{tabular}{lcc}
\hline Grupo & Macho & Hembra \\
\hline G1 - E. coli & 14.3 & 23.1 \\
G2 - Salmonella & 23.1 & 33.3 \\
G3 - E. coli - & 45.5 & 45.5 \\
Salmonella & 6.7 & 6.7 \\
G4 - Testigo $^{2}$ & 6.7
\end{tabular}

${ }^{1} 27$ crías (Muertos: 1 macho y 3 hembras) en G1, 25 crías (Muertos: 4 machos y 4 hembras) en G2, 22 crías (Muertos: 5 machos y 5 hembras) en $\mathrm{G} 3$, y 30 crías (Muertos: 1 macho y 1 hembra) en $\mathrm{G} 4$

2 Positivas a E. coli, Salmonella o ambas y tratadas con tetraciclinas

\section{Discusión}

Los resultados de laboratorio indicaron mayor presencia de E. coli, Salmonella spp y la asociación E. coli - Salmonella spp en las crías de alpacas con enteropatías diarreicas en ambos sexos. La elevada prevalencia de estos patógenos se debería al proceso de colonización de las bacterias por su mecanismo de acción de intracelulares facultativos que ocurre en animales de sangre caliente (di Conza et al., 2012) y por el deficiente control sanitario en los rebaños alpaqueros (More et al., 2011), donde se liberan las bacterias al medio ambiente (en especial al suelo, agua y pastos) cuando son expulsadas por vía fecal, constituyendo un riesgo de infección entérica (Rosadio et al., 2012b).

Lucas et al. (2016) identificaron E. coli en el $8 \%$, Salmonella spp en el $18.3 \%$ (104/132) y asociación de bacterias y parásitos en el $23.3 \%$ de 60 crías de alpacas de 1 a 2.5 meses de edad con cuadros mortales de diarrea; siendo estos resultados mayores a los del presente estudio, donde se encontró positivos al 12\% (18/104) de los machos y $11 \%$ de las hembras (17/104). Así mismo, Morales (2017) logró aislar $E$. coli en el $47.8 \%$ (43/90) de alpacas con diarrea y en el 58.3\% $(35 / 60)$ sin diarrea, en tanto que Chuquizuta y Morales (2017) aislaron E. coli en el 40.8\% y Salmonella spp en el $39.3 \%$ de gazapos muertos en una granja de cuyes de crianza intensiva. Las elevadas tasas de prevalencia de E. coli y de Salmonella spp estarían relacionadas al uso innecesario y/o aplicación prolongada de antibióticos, así como a la aplicación de dosis incorrectas (Carhuapoma et al., 2018).

El mayor efecto negativo en el peso corporal encontrado en las crías del grupo G3 (E. coli - Salmonella spp) y G2 (Salmonella spp) se debe a que estas bacterias son enterohemorrágicas; de allí que causan deshidratación progresiva, acidosis, postración, pérdida de peso y muerte (Ortiz, 2011). Además, ocasionan una disfunción intestinal asociada a diarrea y sobreestimulación de la función secretora de las criptas epiteliales (Rosadio et al., 2012b). Los animales pierden el apetito y dejan de consumir la leche materna (Pinto et al., 2010). En condiciones de buena salud, las crías de alpacas alcanzan a tener pesos que oscilan entre 18 y $20 \mathrm{~kg}$ y talla corporal de $80 \mathrm{~cm}$ a los 2 meses de edad (Cuenca, 2012).

El mayor efecto negativo de la asociación E. coli-Salmonella spp podría significar que las cepas enteropatogénicas eae y $b f p$ de $E$. coli estarían asociándose a la capacidad de adherirse a los enterocitos, inicialmente en forma laxa, a través del producto $b f p \mathrm{y}$, posteriormente, en una forma más fuerte que elimina («cepillado») las microvellosidades intestinales a consecuencia de la proteína intimina codificada por el gen eae (Rosadio et al., 2012b). Esto favorecería el cuadro diarreico y alteración de temperatura corporal por estimulación de las criptas epiteliales (Salmonella, colibacilosis) (Mamani et al., 2009). Además, la asociación E. coli - Salmonella spp podrían generar procesos neumónicos en neonatos y crías 
de alpacas (Ortiz, 2011), lo cual generaría una menor ganancia de peso y talla de los animales (Valencia et al., 2015), como se observó en los grupos G2 y G3. Estos resultados concuerdan con el estudio de Cuenca (2012). Por otro lado, las crías de G4 presentaron un desarrollo corporal dentro de los márgenes esperados.

Silvera et al. (2012) llegaron a detectar en cuadros diarreicos de alpacas a las cepas $s t x$-positivas de $E$. coli, las cuales producen infecciones intestinales a través de las verocitotoxinas. Esto estaría contribuyendo a disminuir el apetito $y$, consecuentemente, el menor peso y talla corporal de las crías. Por otro lado, se conoce que las crías hembras serían más sensibles frente a las cepas stxpositivas de E. coli (Pando, 2011). No se dispone de reportes científicos de la influencia negativa de la patogenicidad de Salmonella spp en los procesos fisiopatológicos, metabólicos y de desarrollo en las crías de alpacas (Chiok, 2012; Dionisio, 2012; Lucas et al., 2016), pero se presume que estos procesos estarían afectados por Salmonella spp $\mathrm{y}$, por lo tanto, estarían directamente asociados al bajo peso y talla, así como a la alta mortalidad, tal y como ocurre en otras especies domésticos (Gallegos, 2013).

La alta tasa de mortalidad ocurrida en los grupos que presentaban la asociación $E$. coli-Salmonella spp y solo Salmonella spp podría confirmar a estos agentes dentro de los principales agentes potencialmente productores de diarreas en alpacas neonatas y crías (Mamani et al., 2009; Rosadio et al., 2012a) y estarían asociados a casos esporádicos y brotes de diarrea generando colitis hemorrágica y síndrome urémico hemolítico (Jurea et al., 2015), lo cual ocasiona enfermedad extraintestinal severa, caracterizada por anemia hemolítica microangiopática, trombocitopenia y falla renal aguda (Moredo et al., 2012 ). Las tasas de mortalidad encontradas por enteropatías fueron similares a otros reportes de la literatura científica (Gallegos, 2013; Valencia et al., 2015).

\section{Conclusiones}

- Crías de alpacas con enteropatías diarreicas evidenciaron altos porcentajes de positividad a Escherichia coli, Salmonella spp y a la asociación $E$. coli - Salmonella spp.

- La asociación Escherichia coli Salmonella spp afectó significativamente el peso y talla corporal e incrementó la mortalidad en crías de alpacas con enteropatías diarreicas.

\section{Agradecimiento}

Al Administrador Ejecutivo del Laboratorio Central de Investigación de la Universidad Nacional de Huancavelica y al productor de la Comunidad Campesina de Santa Bárbara por brindar las facilidades para la ejecución del presente estudio.

\section{Literatura Citada}

1. Carhuapoma DV, Mayhua MP, Valencia MN, Lizana HE. 2018. Antibacterial in vitro of effect Urtica dioica and Piper angustifolium in alpacas (Vicugna pacus) with diarrheal enteropathies. MOJ Anat Physiol 5: 160162. doi: 10.15406/mojap.2018.05.00182

2. Chiok R. 2012. Expresión de citoquinas de la respuesta TH1 (Ifnã e Il2) y TH2 (Il4 e Il10) en mucosa intestinal de crías de alpaca (Vicugna pacos) sanas y con enteropatía. Tesis de Maestría. Lima, Perú: Univ. Nacional Mayor de San Marcos. 122 p.

3. Chuquizuta C, Morales S. 2017. Identificación de agentes bacterianos aislados de gazapos muertos de cuyes en una granja de crianza intensiva en Lima, Perú. REDVET 18(12). [Internet]. Disponible en: http://www.veterinaria.org/ revistas/redvet/n121217/121738.pdf

4. Cuenca P. 2012. Caracterización fenotípica y sistema de producción de las alpacas en la Estación Experimental Aña Moyocancha. Tesis de Ing. Zootecnista. Riobamba, Ecuador: Escuela Superior Politécnica de Chimborazo. 109 p. 
5. di Conza JA, Mollerach ME, Gutkind GO, Ayala JA. 2012. Dos aislamientos de Salmonella infantis multirresistentes se comportan como hipoinvasivos, pero con elevada proliferación intracelular. Rev Argent Microbiol 44: 69-74.

6. Dionisio J. 2012. Cinética de expresión de inmunoglobulina A en el epitelio intestinal de crías de alpaca (Vicugna pacos). Tesis de Médico Veterinario. Lima, Perú: Univ. Nacional Mayor de San Marcos. 61 p.

7. Gallegos R. 2013. Índices productivos de alpacas del Centro de Investigación y Producción «La Raya». Rev Investig Alto Andina 15: 255-262.

8. Jurea MA, Condorí MS, Pérez G, Catalána MG, López A, Zolezzi G, Chinen I, et al. 2015. Aislamiento y caracterización de Escherichia coli $\mathrm{O} 157$ en productos cárnicos bovinos y medias reses en la provincia de Tucumán. Rev Argent Microbiol 47: 125-131.

9. Lucas JR, Morales S, Barrios M, Rodríguez J, Vásquez M, Lira B, Torres $B$, et al. 2016. Patógenos involucrados en casos fatales de diarrea en crías de alpaca de la Sierra Central del Perú. Rev Inv Vet Perú 27: 169-175. doi: 10.15381/rivep.v27i1.11465

10. Mamani J, Condemayta $Z$, Calle L. 2009. Causas de mortalidad de alpacas en tres principales centros de producción ubicados en puna seca y húmeda del departamento de Puno. REDVET 10(8). [Internet]. Disponible en: http:// www.veterinaria.org/revistas/redvet/ n080809/080904.pdf

11. Mamani L, Huanca T. 2011. Manual de sanidad en rebaño mixto. Lima: Heifer Perú. $140 \mathrm{p}$.

12. More J, Manchego A, Sandoval N, Ramírez M, Pezo D, Chiok K, Rivera H. 2011. Detección genómica y expresión de péptidos antimicrobianos $(\alpha-\mathrm{y}$ $\beta$-defensinas) en mucosa intestinal de crías de alpaca (Vicugna pacos). Rev Inv Vet Perú 22: 324-335. doi: 10.15381/ rivep.v22i4.332.
13. Moredo FA, Cappuccio JA, Insarralde L, Perfumo CJ, Quiroga MA, Leotta GA. 2012. Caracterización genotípica de aislamientos de Escherichia coli obtenidos de cerdos con diarrea posdestete y enfermedad de los edemas. Rev Argent Microbiol 44: 85-88.

14. Morales S, Siu E, Ramírez P, Navarro A. 2017. Determinación de serotipos de Escherichia coli aisladas de crías de alpacas (Vicugna pacos) con y sin diarrea en Huancavelica. REDVET 18(9). [Internet]. Disponible en: http:// www.veterinaria.org/revistas/redvet/ n090917/091722.pdf

15. Oha R. 2012. Anatomía patológica de las diarreas infecciosas en crías de alpaca (Lama pacos) en la SAIS Aricoma Ltda. 57. Tesis de Médico Veterinario y Zootecnista. Puno, Perú: Univ. Nacional del Altiplano. $79 \mathrm{p}$.

16. Ortiz S. 2011. Evaluación de algunos métodos de control de la mortalidad en crías de alpaca (Lama pacos) en explotaciones familiares. Tesis de Médico Veterinario. Lima: Univ. Nacional Mayor de San Marcos. 58 p.

17. Pando S. 2011. Evaluación de principales características productivas y reproductivas de alpacas Huacaya en el INIA Santa Ana - Huancayo periodo 2004-2008. Tesis de Ing. Zootecnista. Huancayo, Perú: Univ. Nacional del Centro del Perú. $114 \mathrm{p}$.

18. Pinto CE, Martín C, Cid MD. 2010. Camélidos sudamericanos: estado sanitario de sus crías. Rev Complutense Cienc Vet 4: 37-50.

19. Rosadio A, Yaya K, Véliz P, Quispe T. 2012a. Efecto protector de una vacuna polivalente anticlostridial sobre la mortalidad neonatal en alpacas. Rev Inv Vet Perú 23: 299-306. doi: 10.15381/ rivep.v23i3.912

20. Rosadio R, Maturrano L, Pérez, D, Luna L. 2012b. El complejo entérico neonatal en alpacas andinas. Rev Inv Vet Perú 23: 261-271. doi: 10.15381/rivep.v23i3.908 
21. Silvera E, Perales R, Rodríguez J, López T, Gavidia C, Agapito J, Palacios C. 2012. Presencia de Escherichia coli O157 en crías de alpacas (Vicugna pacos). Rev Inv Vet Perú 23: 98-104. doi: 10.15381/rivep. v23i1.888

22. Siuce J, Manchego A, Sandoval N, More J, Kim-Lam Chiok, Pezo D, Rivera H. 2015. Expresión de defensinas en yeyuno de crías de alpacas (Vicugna pacos) con enteropatías. Rev Inv Vet Perú 26: 317-327. doi: 10.15381/ rivep.v26i2.11093

23. Valencia N, Aquino H, Anccasi A. 2015. Causas de mortalidad en crías de alpacas en centros de producción con manejo tecnológico medio y bajo en el distrito de Huancavelica, Perú. En: VII Congreso Mundial en Camélidos Sudamericano. Puno, Perú. 